\title{
TRANSPLANTE PARCIAL ORTOTÓPICO DE FÍGADO AUXILIAR NA HEPATITE FULMINANTE
}

\author{
Auxiliary Partial Orthotopic Liver Transplantation in Fulminant Hepatitis \\ Daniela Medeiros Calil', André Ibrahim David², Alisson Paulino Trevizol', Eduardo Rullo Maranhão Dias', Felipe Soares Oliveira \\ Fiúza', Gabriel Beligni Campi', Ben-Hur Ferraz-Neto ${ }^{3}$
}

\begin{abstract}
RESUMO
A Hepatite Aguda Grave é uma doença de rápido desenvolvimento que causa falência do fígado com coagulopatia e encefalopatia. Devido à irreversibilidade do quadro, a maioria dos casos deve ser tratada com transplante de fígado. Entretanto, existe dificuldade na obtenção de enxertos hepáticos devido à escassez de doadores falecidos e a necessidade da urgência do transplante. Uma terapia inovadora está sendo utilizada nesses casos: o Transplante Parcial Ortotópico de Fígado Auxiliar (TPOFA). Essa técnica consiste na ressecção de parte do fígado acometido e transplante de um enxerto parcial, permitindo assim a permanência de parte do fígado original, possibilitando sua recuperação e a retirada gradual da imunossupressão. Material e Método: Revisão bibliográfica de artigos na plataforma Mesh-Medline com os descritores: "fulminant hepatitis", "acute liver failure", "liver transplantation" e "auxiliary partial orthotopic liver transplantation" e sítios oficiais e do governo para obtenção de dados epidemiológicos. Resultados: Técnica descrita recentemente com boa sobrevivência dos pacientes e que ainda não foi realizada no Brasil. Dentre as vantagens, há a suspensão da terapia imunossupressora mediante a recuperação do fígado original e a redução dos seus efeitos colaterais; além disso, tem-se a opção de utilizar enxertos de doadores vivos. Conclusão: A experiência em outros países do mundo mostrou a viabilidade do Transplante Parcial Ortotópico de Fígado Auxiliar. A realização por equipes devidamente treinadas e capacitadas representará uma evolução do transplante hepático no Brasil.
\end{abstract}

Descritores: Hepatite; Falência Hepática Fulminante; Transplante de Fígado.

\footnotetext{
Instituição:

${ }^{1}$ Acadêmicos da Faculdade de Ciências Médicas da Santa Casa de São Paulo

${ }^{2}$ Serviço de Transplantes do Hospital das Clínicas da FMUSP

3 Programa de Transplantes do Hospital Israelita Albert Einstein
}

Trabalho dentro da linha de pesquisa aprovada com bolsa PIBIC/CNPq para estudo experimental

\author{
Correspondência: \\ Dr. André Ibrahim David \\ Av. Dr. Arnaldo 455, 3 andar, sala 3206, CEP 01246-903, São Paulo/SP \\ Tel/fax: (11) 3061-7270 \\ E-mail: andredavidmd@gmail.com
}

\section{INTRODUÇÃO}

Hepatite fulminante é uma doença de rápido desenvolvimento que pode causar falência aguda do fígado com perda funcional e o consequente desenvolvimento de coagulopatias e encefalopatia. As principais etiologias são: hepatotoxicidade por ingestão de acetaminofeno (paracetamol), intoxicação por cogumelos (Amanita phalloides), hepatotoxicidade induzida por drogas variadas, hepatite viral, hepatite auto-imune, Doença de Wilson, doença isquêmica do fígado frequente após parada cardíaca (período de intensa hipovolemia e hipotensão), infiltração maligna (metástases de tumores de mama, de pulmão de células pequenas, de linfomas e de melanomas) e causas indeterminadas.

A etiologia da hepatite fulminante varia conforme as regiões geográficas. ${ }^{1}$ Anualmente, há aproximadamente 1,5 milhões de casos de hepatite A aguda em todo o mundo e, dependendo da região, pode chegar à cerca de 150/100.000. As hepatites A e B representam $15 \%$ e $10 \%$ dos casos nos países mediterrâneos, respectivamente, enquanto que $45 \%$ dos casos são de origem indeterminada. Na Inglaterra, a hepatite por paracetamol é a principal causa da doença. Nos Estados Unidos da América, a incidência de hepatite fulminante causada pelos vírus da hepatite A e B em 2007, foi de 1/100.000 e 1,5/100.000, respectivamente. ${ }^{2}$

No ano de 2007 foram realizados 48 transplantes de fígado no Estado de São Paulo devido a casos de hepatite fulminante. No ano anterior, haviam sido feitos 28 transplantes. Esses números correspondem a $11 \%$ do total de transplantes de fígado realizados no Estado. ${ }^{3}$ 
As manifestações clínicas da hepatite fulminante são muito variadas, mas alguns dos sintomas típicos incluem: ascite, icterícia, edema cerebral e hipertensão intracraniana (causando encefalopatia), coagulopatias, sangramentos gastrintestinais, falência renal, alterações metabólicas diversas (acidose, alcalose, hipoglicemia, diminuição de eletrólitos no sangue, etc) e maior susceptibilidade ao desenvolvimento de infecções oportunistas. ${ }^{4,5}$

Os pacientes com hepatite fulminante evoluem com encefalopatia hepática. Essa é provocada pela diminuição da metabolização de toxinas pelo fígado, que permanecem na corrente sanguínea e atingem o SNC. Nessas condições ocorre aumento da pressão intracraniana devido ao edema cerebral e lesão do Sistema Nervoso Central. ${ }^{4}$

Os diversos agentes etiológicos da hepatite fulminante causam alterações morfológicas primariamente idênticas, que variam de acordo com a gravidade do processo necrosante. Em todos os casos, a distribuição do comprometimento hepático pode ser total ou restrito a algumas áreas aleatórias. O fígado pode perder substância maciçamente. Com isso, o órgão torna-se vermelho, flácido e coberto por uma cápsula enrugada. Microscopicamente nota-se a destruição completa dos hepatócitos em lóbulos contíguos, deixando apenas um arcabouço de reticulina colapsado e tratos portais preservados (porém, na necrose centrolobular causada por hepatotoxinas diretas ou isquemia, o arcabouço parenquimatoso é preservado). Pode haver apenas reação inflamatória que, na sobrevida por vários dias, é aumentada por um afluxo maciço de células inflamatórias para o início do processo de fagocitose. ${ }^{6}$

Caso o paciente sobreviva por mais de uma semana, inicia-se uma atividade regenerativa secundária dos hepatócitos e ductos biliares sobreviventes. Os canais de Hering (compartimento proliferativo bipotencial que liga os hepatócitos com a árvore biliar) dão origem a estruturas ductulares mal formadas. Células-tronco adormecidas presentes ao longo dos dúctulos biliares e canais de Hering proliferam-se, dando origem às células ovais (pequenas células com alta razão núcleo-citoplasmática). Se houver tempo suficiente (sobrevida do paciente além das primeiras semanas), o fígado é capaz de recuperar-se completamente com a maturação de todas as populações de células proliferantes, para dar origem a hepatócitos e células epiteliais dos ductos biliares morfologicamente normais. ${ }^{6}$

O tratamento da hepatite fulminante consiste no tratamento de suporte e transplante hepático. O tratamento de suporte consiste em garantir ao paciente uma nutrição adequada, otimizar os balanços hídricos e ventilação mecânica. A monitorização da pressão intracraniana nos indivíduos que apresentam encefalopatia e a remoção de qualquer agente hepatotóxico é fundamental para o sucesso do tratamento nessa fase crítica. Devido à gravidade do quadro, a maioria dos casos de hepatite fulminante requer transplante hepático. Sem a realização do transplante, a mortalidade encontra-se em torno de $95 \% .^{3}$ Assim, existem alguns critérios que são utilizados para determinar quais pacientes devem realizar o transplante: critérios de Clichy e O'Grady .

O critério de Clichy determina que pacientes que apresentam encefalopatia hepática, independente do grau, são candidatos ao transplante hepático. Além disso, pacientes maiores de 30 anos que apresentam Fator V inferior a 30\% ou pacientes menores de 30 anos que apresentam Fator V menor que 20\% também são candidatos ao transplante de fígado.

O critério do King's College (O'Grady) classifica os pacientes em: aqueles que ingeriram acetaminofen e aqueles que não ingeriram.
Indivíduos que ingeriram acetaminofen e apresentam $\mathrm{pH}$ abaixo de 7,3, independentemente do grau de encefalopatia, são candidatos ao transplante hepático. Além disso, pacientes que apresentam Tempo de Protrombina (TP) maior que 100 segundos ou INR maior que 6,5 e creatinina maior que $3,4 \mathrm{mg} / \mathrm{dL}$ e encefalopatia graus III ou IV também são aceitos para transplante de fígado. Já os indivíduos que não ingeriram acetaminofen e apresentam TP maior que 100 segundos ou INR maior que 6,5 (independente do grau de encefalopatia) ou que preencham três dos critérios a seguir: idade menor que 10 ou maior que 40 anos; hepatite causada por vírus $\mathrm{A}$ ou $\mathrm{B}$, halotano, reação idiossincrática a drogas ou etiologia indeterminada; icterícia há mais de sete dias antes do início da encefalopatia; TP maior que 50 segundos ou INR maior que 3,5; concentração sérica de bilirrubina maior que $17,5 \mathrm{mg} / \mathrm{dL}$.

Entretanto, existe a dificuldade na obtenção de enxertos hepáticos para realização do transplante hepático devido à escassez de doadores falecidos e à necessidade da urgência do transplante na hepatite fulminante. Dessa forma, terapias alternativas vêm sendo sugeridas na tentativa de contornar esse problema.

Uma terapia alternativa ao tratamento tradicional é o Transplante Parcial Ortotópico de Fígado Auxiliar (TPOFA). Essa técnica consiste na ressecção de parte do fígado acometido e no transplante de um enxerto parcial, permitindo, assim, a permanência de grande massa do fígado original, possibilitando potencial recuperação futura. Assim sendo, seria possível a retirada gradual da terapia imunossupressora, tornando o paciente menos sujeito aos efeitos colaterais desse tratamento. ${ }^{7,8}$ Apesar da evolução da terapia imunossupressora nos últimos anos diminuindo a rejeição e permitindo maior sobrevida do enxerto, o paciente transplantado apresenta seus efeitos adversos. As principais manifestações são insuficiência renal, osteopenia, dislipidemias, neurotoxidade, hipertensão, toxicidade gastrointestinal, diabetes, infecções oportunistas e desenvolvimento de neoplasias. ${ }^{9}$

No TPOFA, com a regeneração do fígado primário é possível que a imunossupressão seja suspensa, diminuindo os efeitos colaterais para o paciente. É importante ressaltar o benefício dessa suspensão nos transplantes pediátricos, já que estudos revelam que doenças linfoproliferativas pós-transplantes (infecções por vírus Epstein-Barr), decorrentes da imunoterapia são mais comuns em crianças; além disso, o TPOFA evita os efeitos adversos da imunossupressão durante toda a vida.

O TPOFA é um tratamento recente e algumas variáveis do momento da indicação ainda não estão bem estabelecidas. Quando o fígado ainda tem capacidade de regeneração é o principal questionamento. O objetivo deste trabalho é realizar uma revisão da literatura a respeito desse assunto, para que, futuramente, possam ser realizados modelos experimentais e, a partir disso, determinar o momento mais adequado para realização do TPOFA no tratamento da hepatite fulminante. Existem atualmente alguns trabalhos na literatura que descrevem o modelo de hepatite fulminante induzida em suínos e técnicas de transplante de fígado parcial. .10,11 $^{1}$

\section{MATERIAL E MÉTODO}

Para a realização deste trabalho foram levantados artigos na plataforma do Medline, utilizando-se o Mesh. Os seguintes descritores foram utilizados: "fulminant hepatitis", "acute liver failure", "liver transplantation" e "auxiliary partial orthotopic liver transplantation". Como resultado dessa pesquisa foram encontrados 
200 artigos. Desses, 50 artigos descreviam técnicas de transplante de fígado, 15 eram sobre experimentos em suínos ou ratos, 20 sobre experiência clínica em transplante de fígado e 15 sobre hepatite fulminante. Desse conjunto, foram selecionados sete artigos, que descreviam técnicas de indução de hepatite fulminante em porcos, técnicas cirúrgicas para realização do TPOFA em animais de grande porte, transplante de fígado em crianças, relatos de experiências clínicas em outros países, efeitos colaterais da terapia imunossupressora e causas e características da hepatite fulminante.

Além disso, foi utilizado o livro Bases Patológicas das Doenças de Robbins \& Cotran para obtenção de histologia hepática na hepatite fulminante. Finalmente, foram acessados sites do governo para obtenção de dados epidemiológicos a respeito de hepatite fulminante e transplantes de fígado no Brasil e no mundo.

\section{RESULTADOS}

O TPOFA é uma técnica alternativa de transplante de fígado, que apresenta diversos benefícios e já é realizada em centros de transplante. ${ }^{5,8,12}$

Dentre as vantagens do TPOFA podemos citar: a) maior facilidade para se encontrar enxertos tanto para receptores adultos quanto pediátricos, visto que a cirurgia pode ser realizada intervivos, b) suspensão da terapia imunossupressora mediante recuperação do fígado nativo e redução dos efeitos colaterais típicos de imunodeprimidos..$^{5,8,13}$

O TPOFA oferece também uma opção ao problema da escassez de enxertos, uma vez que os enxertos utilizados nesse transplante podem ser doados por parentes do paciente (mãe, pai, irmãos). Em pacientes com hemofilia $\mathrm{A}$, nos quais há deficiência na produção hepática do fator VIII de coagulação sem outras alterações hepáticas, o TPOFA é visto como uma terapia promissora. Uma vez que a hemofilia não é uma doença letal, há grande dificuldade de se obter enxertos de transplantador cadavérico para esses pacientes. Estudos utilizando TPOFA demonstraram aumento de $30 \%$ no fator VIII de coagulação após o transplante, que se manteve mesmo após 6 semanas. ${ }^{14}$

O TPOFA apresentou melhores resultados em pacientes com menos de 40 anos que apresentavam hepatite fulminante ao invés de hepatite sub-fulminante e cuja etiologia era vírus da hepatite $\mathrm{A}$, vírus da hepatite B ou paracetamol. ${ }^{11}$ Além disso, a recuperação do fígado nativo tem maiores chances de ocorrer em pacientes que apresentaram um intervalo pequeno entre a icterícia e a encefalopatia. ${ }^{12}$
Assim como todo procedimento cirúrgico, o TPOFA apresenta desvantagens, dentre as quais podemos citar o maior risco de complicações. Embora tenham taxas de sobrevida semelhantes, o TPOFA apresenta maior risco de complicações vasculares e bacterianas, quando comparado ao transplante de fígado tradicional. Além disso, as taxas de sequelas neurológicas, disfunção primária do enxerto e necessidade de retransplante também se mostraram mais elevadas. ${ }^{15,16} \mathrm{~A}$ incidência de complicações biliares também se mostrou maior no TPOFA do que no transplante tradicional de fígado. Devido a esses fatores, de acordo com estudo realizado na Universidade de Kyoto, o TPOFA deve ter uma indicação restrita à hepatite fulminante, enxertos pequenos para o tamanho e incompatibilidade ABO. ${ }^{12}$

Outro problema observado nos casos em que se optou por realizar TPOFA é que o saldo do fluxo de sangue portal para o fígado nativo e para o enxerto pode mudar radicalmente ao longo do tempo, de acordo com o estado do fígado transplantado. Em alguns casos, a diminuição do fluxo portal do enxerto pelo fenômeno do roubo portal pode levar à disfunção do enxerto e perda do mesmo. É, então recomendada transecção da veia porta nativa no momento do transplante como um método para evitar essa competição funcional. ${ }^{14}$

Como o TPOFA já é realizado em outros países, sua viabilidade já está fundamentada. ${ }^{5,8,13,17}$ Faz-se necessária a realização de experimentos em animais de grande porte, como suínos, por exemplo, para consolidação da técnica., ${ }^{70}$

O número de transplantes realizados anualmente no Brasil tem aumentado constantemente desde a década de 1990; porém, a demanda por enxertos também vem aumentando progressivamente. O TPOFA apresenta uma opção para essa questão, uma vez que existiria a possibilidade de obtenção de enxertos hepáticos de doadores vivos, parentes ou amigos dos pacientes que estivessem dispostos a doar uma parte de seu fígado.

\section{CONCLUSÃO}

A experiência em outros países do mundo mostrou a viabilidade do transplante parcial ortotópico de fígado auxiliar.

A realização do TPOFA por equipes devidamente treinadas e capacitadas, no momento de melhor indicação (ou seja, quando o fígado nativo ainda for capaz de se regenerar) representará uma evolução no transplante hepático no Brasil.

\section{ABSTRACT}

Severe Acute Hepatitis is a disease that causes rapid development of liver failure with coagulopathy and encephalopathy. Due to the irreversibility of this condition, the majority of cases should be treated with liver transplantation. However, it is difficult to obtain liver grafts due to the shortage of cadaveric donors and the need for urgent transplantation. An innovative therapy is being used in these cases: Auxiliary Partial Orthotopic Liver Transplantation. This technique consists of resection of the part affected of the liver and to transplant a partial graft allowing the original liver to recovery and a gradual withdrawal of immunosuppression. Material and Methods: A literature review of articles in the Medline-mesh platform with the terms "fulminant hepatitis", "acute liver failure", "liver transplantation" and "auxiliary partial orthotopic liver transplantation" plus governmental and official sites to obtain data of epidemiological studies. Results: This technique was recently described with good patient survival rates and it has not been performed in Brazil. Among the advantages are the suspension of immunosuppressive therapy due to the recovery of the original liver and reducing its side effects, and also posing the option of using grafts from living donors. Conclusion: Auxiliary Partial Orthotopic Liver Transplantation is a feasible treatment as shown in international transplant centers. It must be done by properly trained and skilled teams and it will represent an evolution in the liver transplantation in Brazil.

Keywords: Hepatitis; Liver Failure, Acute; Liver Transplantation. 


\section{REFERÊNCIAS:}

1. Guia de Vigilância Epidemiológica - 2a reimpressão (2007). Acesso em 08/2010. Disponível em: http://www.medicinanet.com.br/conteudos/biblioteca/2167/ hepatites_virais.htm

2. World Gastroenterology Organization Practice Guidelines. Acesso em 08/2010. Disponível em: http://www.worldgastroenterology.org/assets/downloads/en/pdf/ guidelines/02_acute_hepatitis.pdf

3. Sociedade Brasileira de Infectologia. Acesso em 08/2010. Disponível em: www.infectologia.org.br

4. Polson J, Lee W. AASLD position paper: The Management of Acute Liver Failure. Hepatology. 2005;41:1179-97.

5. Otte JB. Auxiliary partial orthotopic liver transplantation for acute liver failure in children. Pediatric Transplantation. 1999;3:252-6.

6. Crawford JM. Fígado e Trato Biliar. In: Robbins SL, Cotran RS, editores. Bases Patológicas das Doenças. Rio de Janeiro: Elsevier; 2005. p. 944-45.

7. Fukueda M, Ishizaki N, Hamada N, Kadono J, Kaieda M, Nakamura N, et al. Porcine model of Auxiliary Partial Orthotopic Liver Transplantation for acute liver failure. Transplantation. 2006;82:1312-8

8. Bismuth H, Azoulay D, Samuel D, Reynes M, Grimon G, Majno P, et al. Auxiliary partial orthotopic liver transplantation for fulminant hepatitis. Annals of Surgery. 1996;224:712-26.

9. Danovitch G. Immunosuppressant-induced metabolic toxicities. Transplantation Reviews. 2000;14:65-81.
10. Ishiguro S, Takada Y, Gu M, Fukunaga K, Taniguchi H, Seino K, et al. Auxiliary Partial Orthotopic Liver Transplantation for fulminant hepatitis: regeneration of the diseased native liver in a pig model. Transplantation. 2003;75:1901-4.

11. Taniguchi H, Takada Y, Fukunaga K, Yuzawa K, Otsuka M, Todoroki K. Establishment of a swine model for Auxiliary Partial Orthotopic Liver Transplantation. Transplant Proc. 1998;30:3232-6.

12. Kasahara et al. Auxiliary Parital Orthotopic Living Donor Liver Transplantation: Kyoto University Experience. American Journal of Transplantation. 2005;5:558-565.

13. Park YK, Kim BW, Wang HJ, Kim MW. Auxiliary Partial Orthotopic Living Donor Liver Transplantation in a Patient With Wilson's Disease: A Case Report. Transplant Proc. 2008;40:3808-9.

14. Ko S, Tanaka I, Kanehiro H, Kanokogi H, Ori J, Shima M, et al. Preclinical experiment of Auxiliary Partial Orthotopic Liver Transplantation as a curative treatment for hemophilia. Liver Transplantation. 2005;11:579-584.

15. Kobayashi T, Sato Y, Yamamoto S, Takeishi T, Oya H, Hideki N, et al. Auxiliary Partial Orthotopic Living Donor Liver Transplantation for Fulminant Hepatic Failure With Flat Electroencephalogram: A Case Report. Transplantation Proceedings. 2010;42:990-3.

16. Azoulay D, Samuel D, Ichai P, et al. Auxiliary partial orthotopic versus standard orthotopic whole liver transplantation for acute liver failure: a reappraisal from a single center by a case-control study. Annals of Surgery. 2001;234:723-31.

17. Emre S, Arnon R, Cohen E, Morotti R A, Vaysman D, Shneider B L. Resolution of hepatopulmonary syndrome after auxiliary partial orthotopic liver transplantation in abernethy malformation: a case report. Liver Transplantation. 2007;13:1662-68. 